\title{
Corn Yield Response to Pyraclostrobin with Foliar Fertilizers
}

\author{
John Shetley ${ }^{1}$, Kelly A. Nelson ${ }^{2}$, William G. Stevens ${ }^{3}$, David Dunn ${ }^{3}$, Bruce Burdick ${ }^{4}$, Peter P. Motavalli ${ }^{1}$, \\ James T. English ${ }^{5}$, \& Christopher J. Dudenhoeffer ${ }^{2}$ \\ ${ }^{1}$ Department of Soil, Environmental and Atmospheric Sciences, University of Missouri, Columbia, MO, USA \\ ${ }^{2}$ Division of Plant Sciences, University of Missouri, Novelty, MO, USA \\ ${ }^{3}$ Division of Plant Sciences, University of Missouri, Portageville, MO, USA \\ ${ }^{4}$ Hundley-Whaley Center, University of Missouri, Albany, MO, USA \\ ${ }^{5}$ Division of Plant Sciences, University of Missouri, Columbia, MO, USA \\ Correspondence: Kelly A. Nelson, University of Missouri, Lee Greenley Jr. Memorial Research Center, Novelty, \\ MO 63460, USA. Tel: 1-660-739-4410. E-mail: nelsonke@missouri.edu
}

Received: March 25, 2015 Accepted: April 29, 2015 Online Published: June 15, 2015

doi:10.5539/jas.v7n7p18 URL: http://dx.doi.org/10.5539/jas.v7n7p18

\begin{abstract}
Strobilurin fungicides, including pyraclostrobin, protect many crops from several fungal pathogens and create opportunities to increase plant health and yields. However, corn (Zea mays L.) and many other plants' physiological responses to pyraclostrobin include increases in processes that require nutrients. By applying foliar fertilizers, growers can adjust their nutrient-management strategy based on the plant's reaction to pyraclostrobin. Identifying plants' increased nutrient demands and meeting them with a foliar fertilizer at the time of fungicide application (tasselling) could increase yields. This study evaluated effects of foliar-applied pyraclostrobin at 0.11 $\mathrm{kg} \mathrm{ha}^{-1}$ a.i. with or without 13 commonly available foliar fertilizers on yield, tissue macro- and micronutrient concentrations, severity of disease, and grain quality. Field research occurred at three University of Missouri research centers from 2008-2009. One foliar fertilizer, 0-0-30-0, caused up to $20 \%$ crop injury. Diseases affected plants in all six site-years, but overall severity was low $(\leq 2 \%)$ and likely did not impact crop performance. Pyraclostrobin increased ear leaf $\mathrm{B}$ and $\mathrm{Cu}$ concentrations over all site-years seven days after treatment, and decreased $\mathrm{N}$ concentrations at one site-year. Grain yields increased 5\% at two research sites with pyraclostrobin, and one location had increased grain moisture and grain oil at harvest. One foliar fertilizer, 30-0-0-0, increased grain yields by $10 \%$ at two sites compared to the non-treated control. However, foliar fertilizers showed no observable effects on grain quality characteristics, and none of the foliar fertilizers negatively impacted grain yield.
\end{abstract}

Keywords: corn, disease, foliar fertilizer, fungicide, pyraclostrobin, strobilurin

\section{Introduction}

High-yield corn production systems integrate fungicide applications to maximize plants' photosynthetic efficiency. A fungicide class first available in 1996 includes the strobilurin fungicides (Bartlett et al., 2002). Yield has increased up to $1.5 \mathrm{Mg} \mathrm{ha}^{-1}$ in corn hybrids susceptible to foliar pathogens and $0.38 \mathrm{Mg} \mathrm{ha}^{-1}$ in tolerant hybrids with a strobilurin fungicide such as pyraclostrobin (carbamic acid, [2,[[[1-(4-chlorophenyl)-1H-pyrazol-3-yl]oxy]methyl]phenyl]methoxy-, methyl ester) (Newman, 2009; Bartlett et al., 2002). In wheat, strobilurins increased grain yields over non-treated plants and from 1 to $11 \%$ over other classes of fungicides (Oerke et al., 2001).

Several factors account for corn yield increases with pyraclostrobin. The most direct reason is the reduction in numerous foliar diseases (Bartlett et al., 2002). Worldwide, losses in corn production from fungal and bacterial pathogens alone were estimated to be about 9\% from 2001-2003 (Oerke, 2006). Fungal pathogens, including grey leaf spot (Cercospora zea-maydis), caused yield losses above $44 \%$ in Iowa, $69 \%$ in Virginia and complete crop failure on occasion (Ward \& Nowell, 1998; Ward et al., 1999). Fungal pathogens colonize leaf tissue and often damage the leaves themselves. Leaf necrosis can reduce plants' photosynthetic area and efficiency. Fungal infections also reduce overall physiological efficiency by redirecting plant products and photoassimilates from producing and filling grain to fungal development, growth, metabolism, reproduction, plant defense reactions, 
and respiration of the infected tissue (Venancio et al., 2003). The goal for most fungicide applications is to suppress or eliminate foliar infections, especially in the upper canopy, in order to maintain photosynthetic efficiency (Ward \& Nowell, 1998). This is critical because yield is a direct function of photosynthesis. The eight to nine leaves in the upper canopy supply $75-90 \%$ of the photosynthate that the ear requires during grain fill (Allison \& Watson, 1966).

Strobilurins, including pyraclostrobin, are preventative fungicides that work by inhibiting spore germination (Butzen et al., 2005). The active compounds in strobilurins inhibit mitochondrial respiration by binding to the quinol or ubiquinone oxidation site in the cytochrome-bc $c_{1}$ complex (Bartlett et al., 2002). Binding at this site halts electron transfer in the inner membrane of the mitochondria, which prevents adenosine triphosphate (ATP) from forming and so prevents further energy transfer in the fungal spore (Bartlett et al., 2002; Taiz \& Zeiger, 2006).

Certain physiological alterations in plants may increase grain yield in the absence of disease (Bartlett et al., 2002). A decrease in the progression of senescence with increasing concentrations of pyraclostrobin in spinach leaves has been observed (Grossmann \& Retzlaff, 1997). Maximum retardation of senescence showed $82 \%$ higher chlorophyll levels than the control. Although a delay in senescence could result in a longer duration of grain fill, it could also prolong dry-down and result in higher grain moisture. Glaab and Kaiser (1998) showed that exposure of spinach to kresoxim-methyl (methyl (E)-methoxyimino[2-(o-tolyloxymethyl)phenyl]acetate) increased nitrate reductase activity in the treated leaves even under lighted conditions. Köhle et al. (2002) showed an increase in nitrate assimilation and subsequent yield increase in wheat (Triticum aestivum L.) plants sprayed with pyraclostrobin when compared to a non-treated control. Further grain analysis showed similar C:N ratios, and relative protein contents of treated and untreated plants offered evidence that increased nitrate assimilation in plants contributed to increased growth (Köhle et al., 2002). If protein concentrations and C:N ratios are similar with increased nitrate assimilation, then the whole plant should have increased growth to compensate for the extra nitrate. Increased activity of certain nutrient-containing processes would logically result in greater demand for specific nutrients.

Several studies have established a link between plant nutrition and severity of disease. Plant nutrients such as $\mathrm{Ca}$, $\mathrm{K}, \mathrm{Cl}, \mathrm{Mn}, \mathrm{B}$, and P are important for reducing disease severity in some instances (Brennan, 1992; Rupe et al., 2000; Sweeney et al., 2000; Sanogo \& Yang, 2001; Thomason et al., 2001; Fixen et al., 2004). When combined with a soil fertility program that builds up and maintains optimal soil test nutrient levels, foliar-applied nutrients may directly and positively manage disease. For example, potassium chloride foliar sprays reduce symptoms of several fungal foliar pathogens on several cereal grain crops (Kettlewell et al., 2000). Foliar fertilizers may work in many circumstances leading to the availability of critical nutrients for optimum plant health and productivity.

Combining a foliar fertilizer with fungicide application may reduce application costs, improve disease suppression and nutrient response, and increase flexibility in managing crop response to environmental conditions during the growing season. Although producers cannot rely solely on foliar applications for fertilizer management (Curley, 1994; Johnson et al., 2001, Fernandez et al., 2006; Nelson et al., 2010), foliar fertilizers can complement soil amendments or provide an increase in nutrient supply during plants' peak demand times or when soil conditions restrict nutrient uptake (Fernandez \& Eichert, 2009). Similarly, applying foliar fertilizers to match nutrient demands from physiological responses to pyraclostrobin may result in a synergistic yield increase. Pyraclostrobin, which has a range of fungicidal activity, is labeled for a variety of crops (Bartlett et al., 2002) and received a label for plant health benefits (BASF, 2010). Strobilurin fungicides continue to grow in popularity and usefulness. Combining pyraclostrobin and foliar fertilizers has great potential, not only for its possible synergistic benefit, but also the heightened flexibility it offers producers responding to conditions during the growing season. The objective of this research was to evaluate the effect of common foliar fertilizers in the presence and absence of pyraclostrobin on the severity of disease, ear leaf nutrient concentrations, grain quality, and yield.

\section{Methods}

\subsection{Site Descriptions and Experimental Design}

This study was conducted during 2008 and 2009 at three University of Missouri research centers near Novelty, Portageville, and Albany, Missouri. These locations offer differences in soil and climate, and they represent three of the state's major cropping areas. The diversity of temperature, rainfall, humidity, and soils offers different challenges and issues related to high-yield corn production.

The Greenley Memorial Research Center is located near Novelty in Northeastern Missouri $\left(40^{\circ} 2^{\prime} \mathrm{N}, 92^{\circ} 14^{\prime} \mathrm{W}\right)$; Fisher Delta Research Center is near Portageville in Southeast Missouri $\left(36^{\circ} 25^{\prime} \mathrm{N}, 89^{\circ} 42^{\prime} \mathrm{W}\right)$; and 
Hundley-Whaley Center is in Northwest Missouri $\left(40^{\circ} 15^{\prime} \mathrm{N}, 94^{\circ} 19^{\prime} \mathrm{W}\right)$ near Albany. All three locations were managed for high yielding corn, including two where irrigation was added. Irrigation not only provided adequate water to eliminate yield loss from drought stress, but also provided differing environmental conditions that could affect corn diseases. Novelty had supplemental sprinkler irrigation, while Portageville was flood-irrigated. Albany was not irrigated. Irrigation events were scheduled using the Woodruff chart (Henggeler, 2008). In addition to irrigation, fertility and pest management were targeted at producing high yields. Soil type, management practices, planting and harvest dates, and environmental conditions at the time of foliar fungicide and fertilizer application are reported in Table 1.

Table 1. Field information and management practices at each location in 2008 and 2009.

\begin{tabular}{|c|c|c|c|c|c|c|}
\hline \multirow[t]{2}{*}{ Field information and management practices } & \multicolumn{2}{|c|}{$\begin{array}{c}\text { Novelty } \\
\text { (Knox County) }\end{array}$} & \multicolumn{2}{|c|}{$\begin{array}{c}\text { Portageville } \\
\text { (Pemiscot County) }\end{array}$} & \multicolumn{2}{|c|}{$\begin{array}{c}\text { Albany } \\
\text { (Gentry County) }\end{array}$} \\
\hline & 2008 & 2009 & 2008 & 2009 & 2008 & 2009 \\
\hline Soil series & \multicolumn{2}{|c|}{ Putnam silt loam ${ }^{\dagger}$} & \multicolumn{2}{|c|}{ Tiptonville sandy loam } & \multicolumn{2}{|c|}{ Grundy silt loam ${ }^{\S}$} \\
\hline Previous crop & Corn & Corn & Soybean & Corn & Soybean & Soybean \\
\hline Planting date & 19 May & 7 May & 1 May & 23 Apr. & 21 May & 22 May \\
\hline Replications & 5 & 4 & 4 & 4 & 3 & 5 \\
\hline Fertilizer rate $\left(\mathrm{N}-\mathrm{P}_{2} \mathrm{O}_{5}-\mathrm{K}_{2} \mathrm{O} \mathrm{kg} \mathrm{ha}{ }^{-1}\right)$ & $260-80-110$ & $260-90-130$ & $180-0-0$ & $200-0-0$ & $180-70-90$ & $250-70-90$ \\
\hline Hybrid & DK63-42 & DK63-42 & P33N58 & P33N58 & DK62-43 & DK62-43 \\
\hline Seeding rate (seeds ha $\left.{ }^{-1}\right)$ & 86,500 & 86,500 & 86,500 & 86,500 & 69,200 & 72,900 \\
\hline Fungicide and foliar fertilizer application date & 23 July & 28 July & 9-10 July & 7 July & 16 July & 3 Aug. \\
\hline Air temperature $\left({ }^{\circ} \mathrm{C}\right)$ & 26 & 21 & 24 & 26 & 32 & 31 \\
\hline Relative humidity (\%) & 50 & 90 & 80 & 72 & 70 & 65 \\
\hline Corn height (m) & 2.45 & 1.83 & 3.05 & 2.13 & 3.05 & 2.64 \\
\hline Harvest date & 10 Oct. & 3 Nov. & 22 Sep. & 23 Oct. & 21 Nov. & 3 Nov. \\
\hline
\end{tabular}

Note. ${ }^{\dagger}$ fine, smectitic, mesic Vertic Albaqualfs; ${ }^{\dagger}$ fine-silty, mixed, thermic Typic Argiudolls; ${ }^{\S}$ fine, montmorillonitic, mesic Aquic Argiudolls.

Treatments consisted of a factorial arrangement of the 13 foliar fertilizers (Table 2) and fungicide (presence or absence of pyraclostrobin at $0.11 \mathrm{~kg}$ ai $\mathrm{ha}^{-1}$ ) organized in a random complete block design with three to five replications depending on site and year (Table 1). Combinations of foliar fertilizer and fungicide also included a nonionic surfactant $\left(\right.$ Induce ${ }^{\circledR}$, proprietary blend of alkyl aryl polyoxylkane ethers, free fatty acids, and dimothyl polysiloxane, Helena Chemical Company, Collierville, TN) at $0.25 \%$ vol./vol. All treatments simulated aerial application using a hand boom propelled by $\mathrm{CO}_{2}$ (Bellspray, Inc. Opelousas, LA) at $28 \mathrm{~L} \mathrm{ha}^{-1}$. All applications were made when corn was at VT (tasseling) (Ritchie et al., 1993), which is congruent with typical preventative fungicide application (BASF, 2010). Foliar fertilizers and application rates were selected based on local availability and experience. Table 2 summarizes foliar fertilizer products and application rates. Plots were maintained weed-free as detailed in Table 3 by individual sites and years. 
Table 2. Foliar fertilizer characteristics, manufacturers, and application rates

\begin{tabular}{|c|c|c|}
\hline Fertilizer $\left(\% \mathrm{~N}-\% \mathrm{P}_{2} \mathrm{O}_{5}-\% \mathrm{~K}_{2} \mathrm{O}-\% \mathrm{~S}\right)$ & Trade name and manufacturer & $\begin{array}{l}\text { Application } \\
\text { amount }\end{array}$ \\
\hline $3-18-18-0$ & NA-CHURS/ALPINE Solutions, Marion, OH & $18.7 \mathrm{~L} \mathrm{ha}^{-1}$ \\
\hline $0-0-30-0$ & Double-OK, NA-CHURS/ALPINE Solutions, Marion, $\mathrm{OH}$ & $18.7 \mathrm{~L} \mathrm{ha}^{-1}$ \\
\hline Potassium thiosulfate $(0-0-25-17)$ & KTS, Tessenderlo Kerley Inc., Phoenix, AZ & $9.4 \mathrm{~L} \mathrm{ha}^{-1}$ \\
\hline Potassium thiosulfate plus urea triazone $(5-0-20-13)$ & Trisert K+, Tessenderlo Kerley Inc., Phoenix, AZ & $14.0 \mathrm{~L} \mathrm{ha}^{-1}$ \\
\hline Potassium chloride $(0-0-62-0)$ & PCS, Potash Corp. of Saskatchewan, Northbrook, IL & $2.8 \mathrm{~kg} \mathrm{ha}^{-1}$ \\
\hline $\begin{array}{l}25-0-0-0 \text { controlled release nitrogen as methylene urea and } \\
\text { diurea }\end{array}$ & CoRoN, Helena Chemical Co., Collierville, TN & $28.1 \mathrm{~L} \mathrm{ha}^{-1}$ \\
\hline 24-0-1-0.6 slow release $\mathrm{N}$ with $0.25 \% \mathrm{~B}$ & Pacer N, Crop Production Services, Galesburg, IL & $28.1 \mathrm{~L} \mathrm{ha}^{-1}$ \\
\hline $22-0-2-1$ with $0.25 \% \mathrm{~B}$ & Task Force Maize, Crop Production Services, Galesburg, IL & $9.4 \mathrm{~L} \mathrm{ha}^{-1}$ \\
\hline $30-0-0-0$ & Nitamin, Koch Agronomic Services, Wichita, KS & $9.4 \mathrm{~L} \mathrm{ha}^{-1}$ \\
\hline Boron $(10 \%)$ & NA-CHURS/ALPINE Solutions, Marion, $\mathrm{OH}$ & $2.34 \mathrm{~L} \mathrm{ha}^{-1}$ \\
\hline Mn-chelate & NA-CHURS/ALPINE Solutions, Marion, OH & $2.34 \mathrm{~L} \mathrm{ha}^{-1}$ \\
\hline Fe-Mo-Mn-B-Zn (0.3\%-0.01\%-3.2\%-0.2\%-2.1\%) premix & MAX-IN, Winfield Solutions, LLC, St. Paul, MN & $2.34 \mathrm{~L} \mathrm{ha}^{-1}$ \\
\hline $6-0-0-0$ with $10 \% \mathrm{Ca}$ & Nutri-Cal, CSI Chemical Corp, Bondurant, IA & $23.4 \mathrm{~L} \mathrm{ha}^{-1}$ \\
\hline
\end{tabular}

Table 3. Crop protection chemical applications and rates for all six site-years

\begin{tabular}{|c|c|c|c|c|}
\hline Site-Year & Herbicide & Rate $\left(\mathrm{kg}\right.$ a.i. ha $\left.{ }^{-1}\right)$ & Timing & Date \\
\hline \multirow[t]{3}{*}{ Novelty 2008} & Atrazine $^{\dagger}$ & 2.39 & Preemergence & May 23 \\
\hline & S-metolachlor ${ }^{\ddagger}$ & 1.89 & & \\
\hline & Mesotrione ${ }^{\S}$ & 0.210 & & \\
\hline \multirow[t]{4}{*}{ Novelty 2009} & Atrazine & 0.845 & Preemergence & May 22 \\
\hline & Mesotrione & 0.226 & & \\
\hline & S-metolachlor & 2.26 & & \\
\hline & Glyphosate (N-(phosphonomethyl)glycine) & 1.06 & Postemergence & June 26 \\
\hline \multirow[t]{4}{*}{ Portageville 2008} & Glyphosate & 1.49 & Preemergence & May 1 \\
\hline & Atrazine & 1.10 & & \\
\hline & Glyphosate & 1.49 & Postemergence & June 6 \\
\hline & Atrazine & 1.10 & & \\
\hline \multirow[t]{4}{*}{ Portageville 2009} & Glyphosate & 1.49 & Preemergence & April 23 \\
\hline & Atrazine & 1.10 & & \\
\hline & Glyphosate & 1.49 & Postemergence & May 28 \\
\hline & Atrazine & 1.10 & & \\
\hline \multirow[t]{3}{*}{ Albany 2008} & Atrazine & 3.40 & Preemergence & May 21 \\
\hline & S-metolachlor & 1.89 & & \\
\hline & Glyphosate & 0.409 & Postemergence & June 16 \\
\hline \multirow[t]{3}{*}{ Albany 2009} & Atrazine & 3.40 & Preemergence & May 22 \\
\hline & S-metolachlor & 1.89 & & \\
\hline & Glyphosate & 0.409 & Postemergence & June 19 \\
\hline
\end{tabular}

Note. ${ }^{\dagger}$ (2-chloro-4-(ethylamino)-6-(isopropylamino)-s-triazine); ${ }^{\ddagger}$ (2-Chloro-N-(2-ethyl-6-methylphenyl)-N-[(1S) -2-methoxy-1-methylethyl]acetamide); ${ }^{\S}$ (2-[4-(methylsulfonyl)-2-nitrobenzoyl]-1,3-cyclohexanedione). 


\subsection{Measurements and Evaluations}

Representative soil samples were taken from each experiment (Table 4) and evaluated to establish baseline levels for multiple essential plant macro- (2008 and 2009) and micro-nutrients (2009). Crop injury was rated on a scale of 0 (no visual injury) to $100 \%$ (complete crop death) 7 to 14 days after treatment (DAT). Severity of foliar disease was rated on a scale of 0 (no disease) to $100 \%$ (complete tissue colonization) 28 to 42 DAT.

Table 4. Soil characteristics for all six site-years

\begin{tabular}{|c|c|c|c|c|c|c|c|c|c|c|c|c|c|c|c|}
\hline \multirow{2}{*}{ Year } & \multirow{2}{*}{ Location } & \multirow{2}{*}{$\begin{array}{l}\mathrm{pH}_{\mathrm{s}} \\
\left(0.01 \mathrm{M} \mathrm{CaCl}_{2}\right)\end{array}$} & \multirow{2}{*}{ Organic matter } & \multirow{2}{*}{$\mathrm{CEC}$} & \multirow{2}{*}{ Bray IP } & \multicolumn{10}{|c|}{ Exchangeable $\left(1 \mathrm{M} \mathrm{NH}_{4} \mathrm{AO}_{\mathrm{c}}\right)$} \\
\hline & & & & & & $\mathrm{Ca}$ & $\mathrm{K}$ & $\mathrm{Mg}$ & S & $\mathrm{Zn}$ & $\mathrm{Fe}$ & $\mathrm{Mn}$ & $\mathrm{Cu}$ & $\mathrm{Cl}$ & B \\
\hline & & & $\mathrm{g} \mathrm{kg}^{-1}$ & $\mathrm{cmol}_{\mathrm{c}} \mathrm{kg}^{-1}$ & $\mathrm{~kg} \mathrm{ha}^{-1}$ & ------ & ------ & - & - & $-\mathrm{kg} \mathrm{h}$ & $a^{-1}--$ & ---- & --- & ---- & ------ \\
\hline \multirow[t]{3}{*}{2008} & Novelty & 6.0 & 20.0 & 14.9 & 39.2 & 4033 & 323 & 411 & $-^{\dagger}$ & - & - & - & - & - & - \\
\hline & Portageville & 6.2 & 13.0 & 9.7 & 38.1 & 3418 & 218 & 212 & - & - & - & - & - & - & - \\
\hline & Albany & 5.8 & 26.0 & 18.8 & 69.4 & 5863 & 262 & 780 & - & - & - & - & - & - & - \\
\hline \multirow[t]{3}{*}{2009} & Novelty & 5.0 & 25.6 & 18.4 & 68 & 4239 & 531 & 517 & 6.1 & 0.6 & 127 & 23 & 1.2 & 4.1 & 0.56 \\
\hline & Portageville & 5.6 & 12.8 & 11.0 & 101 & 3164 & 190 & 477 & 6.1 & 0.44 & 51 & 26 & 1.2 & 4.5 & 0.30 \\
\hline & Albany & 5.7 & 29.5 & 19.1 & 66 & 5870 & 294 & 751 & 6.5 & 1.25 & 64 & 39 & 1.6 & 3.2 & 0.45 \\
\hline
\end{tabular}

Note. ${ }^{\dagger}$ Data were not collected in 2008.

Corn ear leaf samples $\left(10 \mathrm{plot}^{-1}\right)$ were collected, dried at 60 to $70{ }^{\circ} \mathrm{C}$ in a forced air oven, ground in a Wiley Mill (Thomas Scientific, Swedesboro, NJ), and plant tissue nutrient concentrations determined for all treatments seven DAT. For elemental analysis, plant tissue was digested (Hach Digesdahl Digestion Apparatus, Hach Company, Loveland, $\mathrm{CO}$ ) using $\mathrm{H}_{2} \mathrm{SO}_{4}$ and $\mathrm{H}_{2} \mathrm{O}_{2}$. Nitrogen concentrations were determined colorimetrically (Laverty, 1963; Keeney \& Nelson, 1982) with a spectrophotometer (Genesys 10, Thermo Spectronic, Rochester, $\mathrm{NY}$ ). Foliar $\mathrm{P}, \mathrm{K}, \mathrm{Ca}, \mathrm{Mg}, \mathrm{Cu}, \mathrm{Fe}, \mathrm{Mn}, \mathrm{Zn}$, and $\mathrm{S}$ concentrations were determined using inductively coupled plasma (ICP) spectroscopy (Mills \& Jones, 1996). For Cl analysis, a $0.15 \mathrm{~g}$ sample was extracted with $30 \mathrm{~mL}$ distilled $\mathrm{H}_{2} \mathrm{O}$ (Brown \& Jackson, 1955). The suspension was agitated on an orbital shaker (Eberbach Corporation, Ann Arbor, MI), filtered (Ahlstrom 642 filter paper), and analyzed using a chloride ion-specific electrode compared to standard solutions. Boron was determined from dry ashed samples, which were dissolved in $1 \mathrm{M}$ $\mathrm{HCl}$, then diluted to $0.1 \mathrm{M} \mathrm{HCl}$, and analyzed with ICP spectroscopy. Corn grain yields were determined from the two center rows with a small-plot combine (Kincaid Equipment, Haven, KS) and adjusted to $150 \mathrm{~g} \mathrm{~kg}^{-1}$ moisture prior to statistical analysis. Grain samples from all sites except Albany in 2008 were collected for protein, oil, and starch and analyzed using near infrared spectroscopy (Foss Infratec 1241, Eden Prairie, MN).

Unless otherwise specified, data were subjected to an analysis of variance and means separated using Fisher's Protected LSD at $P \leq 0.01$. Data were subjected to an $F$ Max test for homogeneity (Kuehl, 1994) and combined over site-years when appropriate. Main effects were presented in the absence of significant interactions (SAS, 2010).

\section{Results and Discussion}

\subsection{Precipitation and Irrigation}

Precipitation amounts for the six site-year growing seasons, from 1 April to 31 November, varied widely. Most were over the ten-year average for the specific site, but one site was below average. Novelty experienced greater-than-average rainfall during both growing seasons. In 2008, rainfall totaled $1182 \mathrm{~mm}, 48 \%$ greater than the 10-year average of $796 \mathrm{~mm}$, and in 2009 rainfall totaled $1087 \mathrm{~mm}, 36 \%$ greater than the average. Sprinklers irrigated the Novelty site both years. In 2008, rainfall and the $149 \mathrm{~mm}$ of irrigation water combined for $67 \%$ more water than average on the field (Figure 1). In 2009, rainfall plus $188 \mathrm{~mm}$ of irrigation water made the total $60 \%$ greater than average. In 2008, Portageville experienced $601 \mathrm{~mm}$ of rain, which was $20 \%$ below the 10 -year average of $752 \mathrm{~mm}$. In 2009, $971 \mathrm{~mm}$ of precipitation fell, resulting in a $29 \%$ increase over the average. Both years had flood irrigation amounts totaling approximately $610 \mathrm{~mm}$ (Figure 2). Total water was $61 \%$ and $110 \%$ greater than the 10-year average in 2008 and 2009, respectively. Albany had higher-than-average precipitation both years. In 2008, precipitation was $861 \mathrm{~mm}, 28 \%$ greater than the 10 -year average of $670 \mathrm{~mm}$. The following year, $863 \mathrm{~mm}$ of rain fell for a $28 \%$ increase over the average. No supplemental irrigation was given at Albany (Figure 3). 

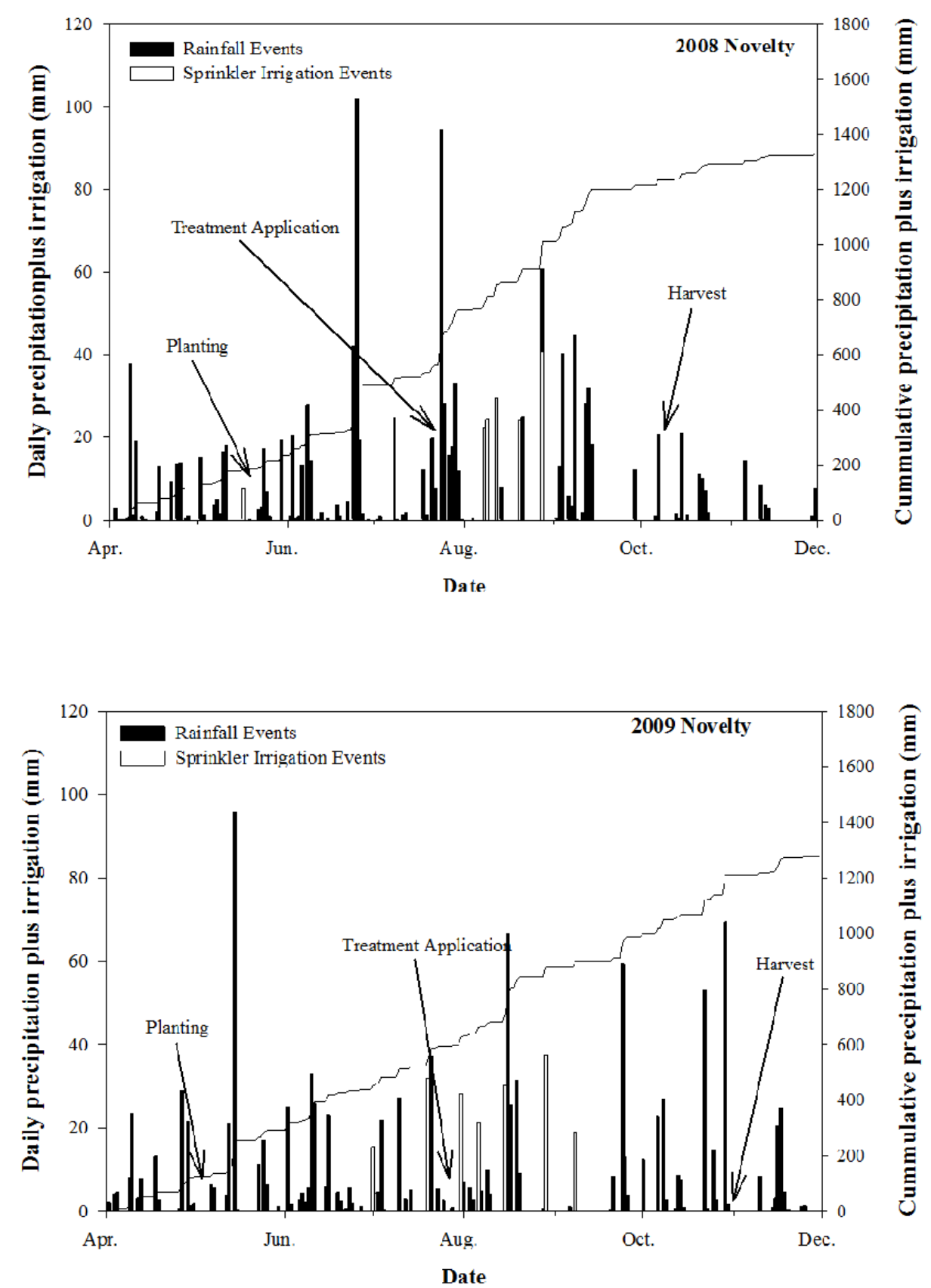

Figure 1. Precipitation and irrigation events, as well as planting, treatment application, and harvest dates at Novelty in 2008 and 2009 

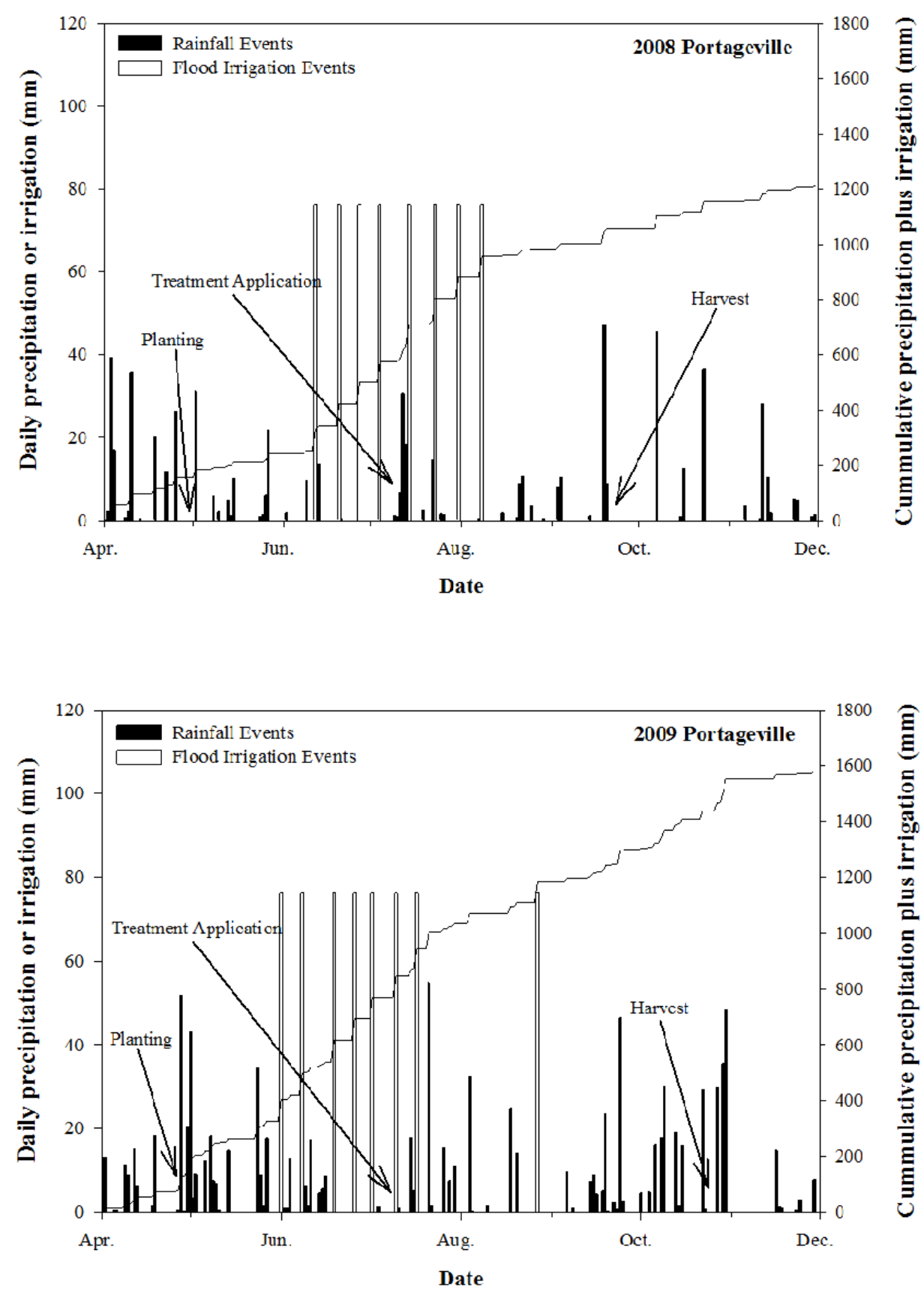

Figure 2. Precipitation and irrigation events, as well as planting, treatment application, and harvest dates at Portageville in 2008 and 2009 

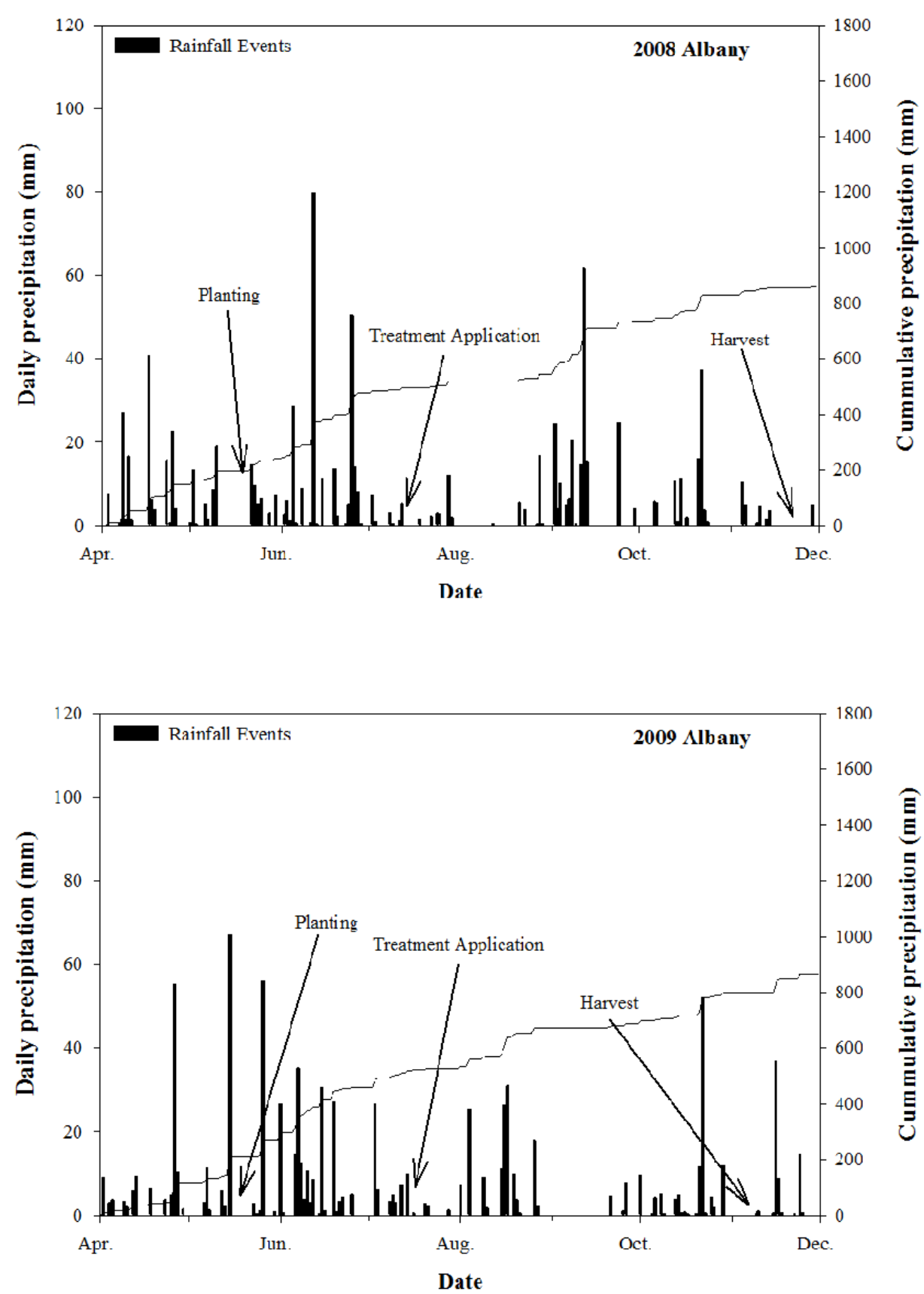

Figure 3. Precipitation, planting, treatment application, and harvest dates at Albany in 2008 and 2009. No supplemental irrigation was provided

\subsection{Crop Injury}

Crop injury, which occurred at all locations over both years, was generally persistent necrosis of leaf tissue depending on the treatment. Pyraclostrobin did not affect crop injury when compared to fertilizer-only treatments even though the treatments included surfactant (Table 5). Some fertilizer treatments caused injury. Overall and for all fertilizer treatments, crop injury was less than $10 \%$ at both Novelty and Albany for both years and less than $20 \%$ at Portageville (Table 6$)$. The only treatment with consistently significant $(P \leq 0.05)$ injury $(4$ to $20 \%)$ for all site-years was 0-0-30-0. The form of potassium in 0-0-30-0 is $\mathrm{K}_{2} \mathrm{CO}_{3}$ derived from potassium hydroxide (Nachurs Alpine Solutions, 2009). At high use rates, this fertilizer can cause necrosis to soybean (Nelson et al., 2012). When 0-0-30-0 was added to glyphosate, the $\mathrm{pH}$ of the solution was $12.0-13.0$, which most likely elevated the injury levels (Nelson et al., 2012). Excluding 0-0-30-0, crop injury was inconsistent over the six 
site-years and was generally less than $10 \%$. Inconsistencies most likely arose from varied environmental conditions during application, such as time of day, temperature, and relative humidity. Hotter and/or dryer conditions may have caused sprays to dry quicker on foliar surfaces, causing increased salt concentrations and leading to greater cellular damage (Neumann \& Prinz, 1975; Gamble \& Emino, 1987).

Table 5. Pyraclostrobin main effects for crop injury 7 to 14 days after treatment, grain yield, and quality characteristics averaged over fertilizer treatments and years (Albany) or site-years (Novelty and Portageville)

\begin{tabular}{|c|c|c|c|c|c|c|c|c|c|c|c|c|}
\hline \multirow{3}{*}{ Fungicide treatment } & \multicolumn{7}{|c|}{ Novelty and Portageville } & \multirow{2}{*}{\multicolumn{5}{|c|}{ Albany }} \\
\hline & \multirow[b]{2}{*}{ Injury } & \multirow[b]{2}{*}{ Yield } & \multicolumn{2}{|c|}{ Grain moisture } & \multirow[b]{2}{*}{ Protein } & \multicolumn{2}{|c|}{ Oil } & & & & & \\
\hline & & & Novelty & Portageville & & Novelty & Portageville & Injury & Yield & Moisture & $\begin{array}{l}\text { Protein } \\
(2009)\end{array}$ & $\begin{array}{l}\text { Oil } \\
(2009)\end{array}$ \\
\hline & $\%$ & $\mathrm{Mg} \mathrm{ha}^{-1}$ & $\mathrm{~g} \mathrm{~kg}^{-1}$ & $\mathrm{~g} \mathrm{~kg}^{-1}$ & $\mathrm{~g} \mathrm{~kg}^{-1}$ & $\mathrm{~g} \mathrm{~kg}^{-1}$ & $\mathrm{~g} \mathrm{~kg}^{-1}$ & $\%$ & $\mathrm{Mg} \mathrm{ha}^{-1}$ & $\mathrm{~g} \mathrm{~kg}^{-1}$ & $\mathrm{~g} \mathrm{~kg}^{-1}$ & $\mathrm{~g} \mathrm{~kg}^{-1}$ \\
\hline Non-treated & 1 & 10.7 & 226 & 162 & 80.4 & 43.1 & 42.2 & 0.5 & 10.5 & 179 & 90.4 & 41.3 \\
\hline Pyraclostrobin & 1 & 11.2 & 231 & 162 & 81.2 & 44.0 & 42.4 & 0.5 & 10.5 & 179 & 90.8 & 41.5 \\
\hline $\operatorname{LSD}(P \leq 0.01)$ & NS & 0.4 & 3 & NS & NS & 0.9 & NS & NS & NS & NS & NS & NS \\
\hline
\end{tabular}

Table 6. Foliar fertilizer main effects for crop injury, grain yield, and grain quality characteristics. Data were averaged over fungicide treatments and years unless designated otherwise

\begin{tabular}{|c|c|c|c|c|c|c|c|c|c|c|c|c|c|}
\hline \multirow{3}{*}{ Fertilizer treatment } & \multicolumn{4}{|c|}{ Injury } & \multirow{2}{*}{\multicolumn{4}{|c|}{ Novelty and Portageville }} & \multirow{2}{*}{\multicolumn{5}{|c|}{ Albany }} \\
\hline & \multicolumn{2}{|c|}{ Novelty } & \multicolumn{2}{|c|}{ Portageville } & & & & & & & & & \\
\hline & 2008 & 2009 & 2008 & 2009 & Protein & Oil & Moisture & Yield & Injury & $\begin{array}{l}\text { Protein } \\
(2009)\end{array}$ & $\begin{array}{l}\text { Oil } \\
(2009)\end{array}$ & Moisture & Yield \\
\hline & \multicolumn{4}{|c|}{ 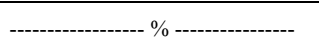 } & \multicolumn{3}{|c|}{--------------g kg ${ }^{-1}$----------- } & $\mathrm{Mg} \mathrm{ha}^{-1}$ & $\%$ & \multicolumn{3}{|c|}{---------------g kg } & $\mathrm{Mg} \mathrm{ha}^{-1}$ \\
\hline Non-treated & 0 & 0 & 0 & 0 & 80.7 & 43.1 & 197.0 & 10.8 & 0 & 91.0 & 41.5 & 179 & 10.6 \\
\hline $3-18-18-0$ & 0 & 0 & 0 & 10 & 79.3 & 42.4 & 196.3 & 11.0 & 0 & 90.8 & 42.0 & 179 & 10.3 \\
\hline $0-0-30-0$ & 7 & 9 & 20 & 10 & 80.8 & 43.0 & 198.3 & 10.1 & 4 & 91.2 & 41.1 & 183 & 10.5 \\
\hline $22-0-2-1,0.25 \% \mathrm{~B}$ & 0 & 0 & 0 & 0 & 80.9 & 43.2 & 196.9 & 11.0 & 2 & 89.9 & 40.9 & 181 & 10.7 \\
\hline $24-0-1-0.6,0.25 \% \mathrm{~B}$ & 0 & 0 & 0 & 0 & 80.4 & 43.1 & 200.6 & 11.0 & 0 & 90.4 & 40.9 & 180 & 10.7 \\
\hline $25-0-0-0,0.01 \% \mathrm{Cl}$ & 0 & 0 & 0 & 10 & 81.3 & 44.1 & 195.4 & 10.9 & 0 & 90.6 & 42.0 & 181 & 11.0 \\
\hline $0-0-25-17$ & 10 & 0 & 0 & 0 & 81.6 & 43.2 & 195.4 & 10.9 & 0 & 90.4 & 41.8 & 180 & 10.5 \\
\hline $5-0-20-13$ & 9 & 2 & 0 & 10 & 82.5 & 42.3 & 196.5 & 11.1 & 0 & 90.1 & 40.2 & 180 & 10.2 \\
\hline $0-0-62-0$ & 0 & 2 & 0 & 0 & 80.5 & 42.7 & 196.7 & 10.9 & 0 & 90.8 & 40.5 & 179 & 10.5 \\
\hline $30-0-0-0$ & 0 & 0 & 0 & 0 & 80.8 & 42.3 & 197.4 & 11.9 & 0 & 90.8 & 40.9 & 177 & 10.8 \\
\hline $6-0-0-0,10 \% \mathrm{Ca}$ & 1 & 0 & 10 & 10 & 80.5 & 42.3 & 197.8 & 11.0 & 0 & 89.2 & 42.0 & 176 & 10.4 \\
\hline B & 0 & 0 & 0 & 10 & 80.4 & 43.4 & 198.7 & 11.4 & 0 & 90.6 & 41.1 & 179 & 10.4 \\
\hline Fe-Mo-Mn-B-Zn & 0 & 0 & 0 & 0 & 80.5 & 43.0 & 198.4 & 10.7 & 0 & 91.8 & 42.7 & 179 & 9.9 \\
\hline Mn-chelate & 0 & 1 & 0 & 20 & 81.4 & 43.0 & 196.4 & 10.7 & 0 & 91.2 & 41.9 & 177 & 10.2 \\
\hline $\operatorname{LSD}(P \leq 0.01)$ & 1 & 1 & 10 & NS & NS & NS & NS & 0.9 & 1 & NS & NS & NS & 0.8 \\
\hline
\end{tabular}

\subsection{Disease Severity}

Severity of foliar disease was less than $5 \%$ for all six site-years (Tables 7 and 8 ). Diseases varied by location and included: anthracnose leaf blight (Colletotrichum graminicola), bacterial stalk rot (Erwinia dissolvens), common rust (Puccinia sorghi), common smut (Ustilago zeae), gray leaf spot (Cercospora zeae-maydis), and northern corn leaf blight (Exserohilum turcicum). Neither pyraclostrobin, nor any of the foliar fertilizers had any significant effect on disease severity. For all treatments over all site-years, severity was quite low, despite elevated crop injury exhibited with some of the fertilizer treatments, optimal weather conditions for disease, and three of the six site-years having continuous corn (Table 1). The lack of disease may have resulted from using hybrids with good to excellent resistance to diseases present at all locations (Sweets \& Wright, 2008; Poland et 
al., 2009). The disease-suppressing effects of pyraclostrobin and foliar fertilizers were not rigorously tested due to lack of disease, even in the non-treated controls. Therefore, yield response may stem from the effects of fungicide treatments or crop nutrition impacts of the fluid fertilizers.

Table 7. Disease severity ratings from each location combined over 2008 and 2009. Data were combined over fertilizer treatments

\begin{tabular}{|c|c|c|c|c|c|c|c|c|c|}
\hline \multirow{2}{*}{ Fungicide treatment } & \multicolumn{3}{|c|}{ Novelty } & \multicolumn{4}{|c|}{ Portageville } & \multicolumn{2}{|c|}{ Albany } \\
\hline & $\mathrm{GLS}^{\dagger}$ & CR & NCLB & GLS & ANTH & BSR & $\mathrm{CS}$ & GLS & $\mathrm{CR}$ \\
\hline & ------- & ----- & - & - & \% & - n & ----- & ----- & ------ \\
\hline Non-treated & 1 & 0.2 & 0.3 & 1 & 2 & 1 & 1 & 1 & 2 \\
\hline Pyraclostrobin ${ }^{*}$ & 1 & 0.1 & 0 & 1 & 2 & 1 & 1 & 1 & 2 \\
\hline $\operatorname{LSD}(\mathrm{P} \leq 0.05)$ & NS & NS & NS & NS & NS & NS & NS & NS & NS \\
\hline
\end{tabular}

Note. ${ }^{\dagger}$ Abbreviations: ANTH, Anthracnose leaf blight (Colletotrichum graminicola); BSR, bacterial stalk rot (Erwinia dissolvens); CR, common rust (Puccinia sorghi); CS, common smut (Ustilago zeae); GLS, grey leaf spot (Cercospora zeae-maydis); LSD, least significant difference; NCLB, northern corn leaf blight (Exserohilum turcicum); and NS, non-significant; ${ }^{*}$ Pyraclostrobin at $0.110 \mathrm{~kg} \mathrm{ha}^{-1}$ plus non-ionic surfactant at $0.25 \% \mathrm{vol} / \mathrm{vol}$.

Table 8. Disease severity ratings from each location were combined over years and fungicide treatments within a location

\begin{tabular}{|c|c|c|c|c|c|c|c|c|c|}
\hline \multirow{2}{*}{ Fertilizer treatment $^{\dagger}$} & \multicolumn{3}{|c|}{ Novelty } & \multicolumn{4}{|c|}{ Portageville } & \multicolumn{2}{|c|}{ Albany } \\
\hline & GLS & $\mathrm{CR}$ & NCLB & GLS & ANTH & BSR & $\mathrm{CS}$ & GLS & $\mathrm{CR}$ \\
\hline & ----- & 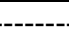 & --- & 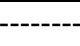 & $\%$ & --- & --- & & ---- \\
\hline Non-treated & 1 & 0.3 & 0 & 1 & 2 & 1 & 1 & 0 & 2 \\
\hline $3-18-18-0$ & 1 & 0.1 & 0 & 1 & 2 & 1 & 1 & 0 & 2 \\
\hline $0-0-30-0$ & 1 & 0.1 & 0 & 2 & 1 & 1 & 1 & 1 & 2 \\
\hline $22-0-2-1,0.25 \% \mathrm{~B}$ & 1 & 0.1 & 0 & 1 & 2 & 1 & 1 & 1 & 2 \\
\hline $24-0-1-0.6,0.25 \% \mathrm{~B}$ & 1 & 0 & 0.1 & 1 & 2 & 1 & 1 & 1 & 3 \\
\hline $25-0-0-0,0.01 \% \mathrm{Cl}$ & 1 & 0.3 & 0 & 1 & 2 & 1 & 1 & 1 & 2 \\
\hline $0-0-25-17$ & 1 & 0.2 & 0 & 1 & 2 & 1 & 1 & 1 & 2 \\
\hline $5-0-20-13$ & 1 & 0.1 & 0 & 1 & 2 & 1 & 1 & 1 & 2 \\
\hline $0-0-62-0$ & 1 & 0.2 & 0 & 1 & 1 & 2 & 1 & 1 & 2 \\
\hline $30-0-0-0$ & 1 & 0.1 & 0 & 1 & 2 & 1 & 1 & 1 & 2 \\
\hline $6-0-0-0,10 \% \mathrm{Ca}$ & 1 & 0.1 & 0.1 & 1 & 2 & 1 & 1 & 1 & 2 \\
\hline Boron & 1 & 0.1 & 0.1 & 2 & 2 & 1 & 1 & 1 & 2 \\
\hline Fe-Mo-Mn-B-Zn & 1 & 0.1 & 0.1 & 1 & 1 & 1 & 1 & 1 & 2 \\
\hline Mn-chelate & 1 & 0.2 & 0 & 1 & 1 & 1 & 1 & 1 & 2 \\
\hline $\operatorname{LSD}(\mathrm{P} \leq 0.05)$ & NS & NS & NS & NS & NS & NS & NS & NS & NS \\
\hline
\end{tabular}

Note. ${ }^{\dagger}$ Abbreviations: ANTH, Anthracnose leaf blight (Colletotrichum graminicola); BSR, bacterial stalk rot (Erwinia dissolvens); CR, common rust (Puccinia sorghi); CS, common smut (Ustilago zeae); GLS, grey leaf spot (Cercospora zeae-maydis); LSD, least significant difference; NCLB, northern corn leaf blight (Exserohilum turcicum); and NS, non-significant.

\subsection{Leaf Nutrient Concentration}

All ear leaf nutrient concentrations in the non-treated control were above the sufficiency levels at the time of application (Mills \& Jones, 1996), with the exception of Ca concentrations (data not presented). Calcium levels were slightly below the sufficiency range. No interactions appeared between fungicide and foliar fertilizer treatment for any nutrient, so data main effects are presented. Interactions with year are presented separately for significant interactions. A significant interaction $(P=0.05)$ occurred between pyraclostrobin treatment and site-year for foliar nitrogen $(\mathrm{N})$ concentrations; therefore, data were presented by individual site-years for ear 
leaf $\mathrm{N}$ concentrations (Table 9). All other foliar nutrient concentrations by pyraclostrobin treatment were averaged over all six site-years (Tables 9 and 10). Only one site-year (Novelty, 2008) showed differences $(P=0.1)$ between the pyraclostrobin-treated and non-treated corn for ear leaf $\mathrm{N}$ concentration. The pyraclostrobin treatment had $6.3 \mathrm{~g} \mathrm{~kg}^{-1}$ lower ear leaf $\mathrm{N}$ concentration. A decrease in tissue $\mathrm{N}$ levels may signify an increase in the activities of certain N-containing physiological processes that can increase with strobilurin fungicides (Glaab \& Kaiser, 1998; Oerke et al., 2001; Köhle et al., 2002).

Table 9. Ear leaf $\mathrm{B}, \mathrm{Cu}$, and $\mathrm{N}$ concentration as affected by pyraclostrobin. Boron and $\mathrm{Cu}$ tissue concentrations were combined over all site-years. Nitrogen foliar concentrations are presented individually by site-year. Data were combined over fertilizer treatments

\begin{tabular}{|c|c|c|c|c|c|c|c|c|}
\hline \multirow{3}{*}{ Fungicide treatment } & \multirow{3}{*}{ Boron } & \multirow{3}{*}{ Copper } & \multicolumn{6}{|c|}{ Nitrogen } \\
\hline & & & \multicolumn{2}{|c|}{ Novelty } & \multicolumn{2}{|c|}{ Portageville } & \multicolumn{2}{|c|}{ Albany } \\
\hline & & & 2008 & 2009 & 2008 & 2009 & 2008 & 2009 \\
\hline & \multicolumn{2}{|c|}{-------'mg kg ${ }^{-1}$} & ------ & - & ------ & $\mathrm{kg}^{-1}$ & 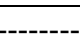 & 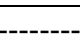 \\
\hline Non-treated & 65.1 & 30.7 & 39.4 & 42.4 & 47.5 & 47.4 & 40.3 & 35.3 \\
\hline Pyraclostrobin & 76.3 & 32.7 & 33.1 & 44.5 & 46.1 & 49.9 & 40.5 & 33.0 \\
\hline $\operatorname{LSD}(P \leq 0.1)$ & 8.3 & 1.9 & 5.6 & NS & NS & NS & NS & NS \\
\hline
\end{tabular}

Table 10. The effect of pyraclostrobin on ear leaf $\mathrm{P}, \mathrm{K}, \mathrm{Ca}, \mathrm{Mg}, \mathrm{S}, \mathrm{Zn}, \mathrm{Fe}, \mathrm{Mn}$, and $\mathrm{Cl}$ concentration. Data were combined over site-years and foliar fertilizer treatments

\begin{tabular}{|c|c|c|c|c|c|c|c|c|c|}
\hline Fungicide treatment & $\mathrm{P}$ & $\mathrm{K}$ & $\mathrm{Ca}$ & $\mathrm{Mg}$ & $\mathrm{S}$ & $\mathrm{Zn}$ & $\mathrm{Fe}$ & $\mathrm{Mn}$ & $\mathrm{Cl}$ \\
\hline & \multicolumn{4}{|c|}{----------g kg ${ }^{-1}$} & \multicolumn{5}{|c|}{ 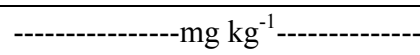 } \\
\hline Non-treated & 2.08 & 22.6 & 1.69 & 2.33 & 290 & 48.8 & 332 & 124 & 5280 \\
\hline Pyraclostrobin & 2.08 & 22.4 & 1.70 & 2.37 & 268 & 47.8 & 334 & 123 & 5270 \\
\hline $\operatorname{LSD}(P \leq 0.1)$ & NS & NS & NS & NS & NS & NS & NS & NS & NS \\
\hline
\end{tabular}

Boron concentrations in corn ear leaf samples increased with pyraclostrobin by $11.2 \mathrm{mg} \mathrm{kg}^{-1}$. Strobilurin fungicides have altered various hormonal processes in plants, and B has been associated with various plant hormonal responses (Venancio et al., 2003; Marschner, 1995). In addition, B has been proposed to exhibit a role in respiration, which can be suppressed by strobilurin fungicides (Parr \& Loughman, 1983; Nason et al., 2007). Strobilurin fungicides have also been shown to retard senescence in plants. Correlated with the impedance of senescence is an increase in indole-3-acetic acid (IAA) concentrations (Marschner, 1995; Grossmann \& Retzlaff, 1997). Boron is closely associated with indole-3-acetic acid metabolism. Altering these biochemical processes with pyraclostrobin may explain differences in the observed $\mathrm{B}$ concentrations in the ear leaf.

Ear leaf $\mathrm{Cu}$ concentrations increased with pyraclostrobin $2.0 \mathrm{mg} \mathrm{kg}^{-1}$. Köhle et al. (2003) showed that plants treated with pyraclostrobin had enhanced tolerance of oxidative stress. Copper is a component of superoxide dismutase, which along with other plant antioxidative enzymes detoxifies superoxide radicals (Taiz \& Zeiger, 2006). Increased $\mathrm{Cu}$ in plant tissue may enhance tolerance of oxidative stress. All other nutrient concentrations showed no significant differences due to the main effect of pyraclostrobin treatment (Table 10).

Foliar fertilizer main effects are presented because limited 2- (year $\times$ fertilizer, fertilizer $\times$ fungicide) and no 3 -way (year $\times$ fertilizer $\times$ fungicide) interactions were observed. A significant year $\times$ fertilizer interaction $(P=$ 0.0009) occurred for ear leaf B concentrations; therefore, data are presented separately by site-year for B (Table 11). No consistent differences were found for ear leaf $B$ concentrations between the non-treated control and the fertilizers that were applied. One foliar fertilizer (6-0-0-0) increased B concentrations over others in two site years. At Novelty in 2009, B concentration increased with 6-0-0-0 $(P=0.1)$ over 25-0-0-0 and Mn-chelate. At Albany that year, B concentration was greater than the non-treated control, 0-0-30-0, and 22-0-2-1. All other significant differences regarding foliar B concentrations were inconsistent and did not seem to show a pattern among site-years or with treatments that included B. 
Table 11. Ear leaf B concentrations by site-year. Data were combined over pyraclostrobin treatments

\begin{tabular}{|c|c|c|c|c|c|c|}
\hline \multirow{3}{*}{ Fertilizer treatment } & \multicolumn{6}{|c|}{ Boron } \\
\hline & \multicolumn{2}{|c|}{ Novelty } & \multicolumn{2}{|c|}{ Portageville } & \multicolumn{2}{|c|}{ Albany } \\
\hline & 2008 & 2009 & 2008 & 2009 & 2008 & 2009 \\
\hline & \multicolumn{6}{|c|}{ 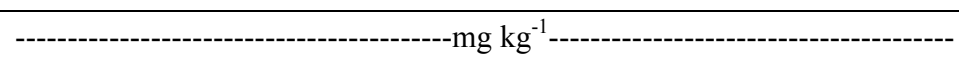 } \\
\hline Non-treated & 53.5 & 26.8 & 45.8 & 49.3 & 72.8 & 54.2 \\
\hline $3-18-18-0$ & 42.8 & 33.0 & 75.8 & 37.0 & 91.2 & 205.8 \\
\hline $0-0-30-0$ & 35.3 & 31.2 & 80.3 & 63.5 & 85.8 & 63.8 \\
\hline $22-0-2-1,0.25 \% \mathrm{~B}$ & 53.5 & 26.8 & 52.2 & 91.3 & 80.2 & 54.0 \\
\hline $24-0-1-0.6,0.25 \% \mathrm{~B}$ & 34.8 & 23.5 & 61.5 & 58.3 & 106.0 & 100.8 \\
\hline $25-0-0-0,0.01 \% \mathrm{Cl}$ & 44.5 & 17.8 & 94.3 & 52.2 & 86.3 & 118.8 \\
\hline $0-0-25-17$ & 46.8 & 27.2 & 48.8 & 48.0 & 81.0 & 135.8 \\
\hline $5-0-20-13$ & 38.8 & 31.8 & 52.2 & 51.0 & 81.8 & 112.7 \\
\hline $0-0-62-0$ & 50.7 & 33.3 & 57.2 & 34.2 & 80.5 & 117.8 \\
\hline $30-0-0-0$ & 67.0 & 33.7 & 53.3 & 59.2 & 90.3 & 218.3 \\
\hline $6-0-0-0,10 \% \mathrm{Ca}$ & 57.0 & 38.7 & 54.3 & 59.0 & 79.8 & 276.5 \\
\hline Boron & 45.3 & 27.3 & 56.5 & 33.7 & 86.2 & 256.0 \\
\hline Fe-Mo-Mn-B-Zn & 41.2 & 29.2 & 75.0 & 33.0 & 85.7 & 136.0 \\
\hline Mn-chelate & 87.7 & 21.2 & 72.5 & 51.3 & 98.0 & 116.8 \\
\hline $\operatorname{LSD}(\mathrm{P} \leq 0.1)$ & 39.6 & 17.4 & 37.7 & 30.5 & 30.5 & 119.0 \\
\hline
\end{tabular}

Other ear leaf nutrient concentrations were averaged over all six site-years (Table 12) because 2- and 3-way interactions were insignificant. With the exception of $\mathrm{Cu}$, all foliar nutrients exhibited significant differences $(P$ $=0.1$ ) among treatments. Ear leaf nutrient concentration with 24-0-1-0.6, 0.25\% B increased over the control and/or other foliar fertilizers for several nutrient concentrations, but had lower ear leaf $\mathrm{S}$ concentrations compared to that of the 5-0-20-13 fertilizer treatment. The fertilizer treatment of $24-0-1-0.6,0.25 \% \mathrm{~B}$ also had a higher $\left(59 \mathrm{mg} \mathrm{kg}^{-1}\right)$ ear leaf Fe tissue concentration compared to the control. All other nutrient concentration increases were greater than the other foliar fertilizers for N, P, and Mg. Surprisingly, 25-0-0-0 increased ear leaf $\mathrm{P}$ concentration $0.19 \mathrm{~g} \mathrm{~kg}^{-1}$ compared to the control. Other differences for foliar nutrient levels were among other foliar fertilizers and were inconsistent. The fertilizer treatment of 5-0-20-13 resulted in the greatest concentrations and an increase over the control for sulfur levels with $369 \mathrm{mg} \mathrm{kg}^{-1}$, a $114 \mathrm{mg} \mathrm{kg}^{-1}$ increase over the control. Conversely, 5-0-20-13 decreased foliar nutrient concentration of $\mathrm{Ca}$ and $\mathrm{Mg}$ compared to the control, and it decreased $\mathrm{P}$ and $\mathrm{K}$ compared to other foliar fertilizers. Ear leaf Mn concentration decreased $16 \mathrm{mg} \mathrm{kg}^{-1}$ with 0-0-62-0 compared to the control, while it decreased $\mathrm{N}, \mathrm{K}, \mathrm{Mg}, \mathrm{Fe}$, and $\mathrm{Mn}$ concentrations compared to several other foliar fertilizers. The micronutrient mix of Fe-Mo-Mn-B-Zn decreased $\mathrm{Mg} 0.20 \mathrm{~g} \mathrm{~kg}^{-1}$ and Zn 5.4 $\mathrm{mg} \mathrm{kg}^{-1}$ compared to the non-treated control, while it decreased $\mathrm{S}$ and Fe concentrations compared to other foliar fertilizers. However, Fe-Mo-Mn-B-Zn increased Mn concentrations compared to other foliar fertilizers, which is expected with $\mathrm{Mn}$ in the formulation. 
Table 12. Ear leaf nutrient concentrations seven days after treatment

\begin{tabular}{|c|c|c|c|c|c|c|c|c|c|c|c|}
\hline Fertilizer treatment & Nitrogen & Phosphorus & Potassium & Calcium & Magnesium & Sulfur & Zinc & Iron & Manganese & Copper & Chloride \\
\hline & \multicolumn{5}{|c|}{ 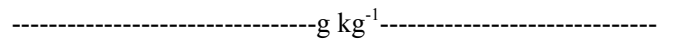 } & \multicolumn{6}{|c|}{ 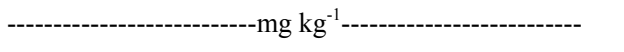 } \\
\hline Non-treated & 41.8 & 2.05 & 23.0 & 1.72 & 2.41 & 255 & 51.1 & 319 & 132 & 30.3 & 5312 \\
\hline $3-18-18-0$ & 39.8 & 2.06 & 23.2 & 1.80 & 2.36 & 256 & 46.3 & 334 & 128 & 29.7 & 4966 \\
\hline $0-0-30-0$ & 42.4 & 2.12 & 23.7 & 1.75 & 2.31 & 258 & 46.6 & 341 & 128 & 32.0 & 5401 \\
\hline $22-0-2-1,0.25 \% \mathrm{~B}$ & 43.1 & 2.03 & 22.6 & 1.68 & 2.37 & 293 & 50.4 & 339 & 125 & 30.2 & 5083 \\
\hline $24-0-1-0.6,0.25 \% \mathrm{~B}$ & 44.5 & 2.19 & 22.7 & 1.74 & 2.47 & 268 & 50.3 & 378 & 131 & 31.1 & 5371 \\
\hline $25-0-0-0,0.01 \% \mathrm{Cl}$ & 41.5 & 2.24 & 22.1 & 1.71 & 2.44 & 265 & 46.6 & 327 & 121 & 31.6 & 5365 \\
\hline $0-0-25-17$ & 44.4 & 2.09 & 23.6 & 1.76 & 2.51 & 336 & 49.6 & 322 & 121 & 31.8 & 5307 \\
\hline $5-0-20-13$ & 43.0 & 1.94 & 21.8 & 1.50 & 2.28 & 369 & 48.3 & 342 & 116 & 32.3 & 5436 \\
\hline $0-0-62-0$ & 43.3 & 2.16 & 22.5 & 1.60 & 2.32 & 258 & 46.8 & 325 & 116 & 32.2 & 5195 \\
\hline $30-0-0-0$ & 42.7 & 2.03 & 22.2 & 1.72 & 2.36 & 263 & 46.9 & 325 & 120 & 31.4 & 5406 \\
\hline $6-0-0-0,10 \% \mathrm{Ca}$ & 40.8 & 2.01 & 22.1 & 1.71 & 2.24 & 249 & 46.7 & 321 & 119 & 32.3 & 5627 \\
\hline Boron & 39.9 & 2.15 & 21.4 & 1.68 & 2.29 & 283 & 49.5 & 334 & 116 & 31.2 & 5216 \\
\hline Fe-Mo-Mn-B-Zn & 41.2 & 2.07 & 22.2 & 1.66 & 2.21 & 243 & 45.7 & 333 & 135 & 34.4 & 5345 \\
\hline Mn-chelate & 43.4 & 2.06 & 22.1 & 1.74 & 2.34 & 250 & 50.8 & 343 & 130 & 32.0 & 4891 \\
\hline $\operatorname{LSD}(P \leq 0.1)$ & 4.17 & 0.17 & 1.65 & 0.18 & 0.18 & 91.9 & 5.20 & 43.7 & 14.5 & NS & 527 \\
\hline
\end{tabular}

Note. Data were combined over site-year and pyraclostrobin treatment.

\subsection{Grain Yield, Moisture, Oil, and Protein Concentration}

In the absence of significant interactions, main effects were presented and data were combined over site-years. Overall, grain yield data for Portageville and Novelty could be combined over all four site-years, whereas Albany data were combined over years. This could be due to differences in seeding rates, presence of irrigation, and/or hybrids. Portageville and Novelty had slightly higher yields than Albany (non-irrigated) probably due to the lack of supplemental irrigation at the Albany site. Pyraclostrobin increased grain yield $0.5 \mathrm{Mg} \mathrm{ha}{ }^{-1}$ at Portageville and Novelty, but showed no effect at Albany (Table 5). Pyraclostrobin did not affect grain moisture content at Albany and Portageville. However, grain moisture at Novelty increased $5 \mathrm{~g} \mathrm{~kg}^{-1}$ with pyraclostrobin compared to the non-treated control (Figure 4). This may be due to differences in environmental conditions at the time of harvest, timing of harvest, or the difference in corn hybrids. However, applying fungicide to corn can increase grain moisture (Bradley \& Ames, 2010; Munkvold et al., 2001). Such increases may result from delayed senescence, similar to reports on other crops with pyraclostrobin and other strobilurin fungicides (Grossman et al., 1999; Ruske et al., 2003; Ypema \& Gold, 1999). This experiment included no measures to support delayed senescence. Similar to wheat studies, pyraclostrobin did not increase grain protein concentration compared to the non-treated control (Köhle et al., 2002). Pyraclostrobin did not increase grain oil concentration at Portageville or Albany; however it increased grain oil concentration $0.9 \mathrm{~g} \mathrm{~kg}^{-1}$ at Novelty (Table 5). Soybean studies have shown similar effects of strobilurin fungicides on grain oil concentrations (Nelson et al., 2010).

Foliar fertilizers did not affect grain protein, oil or moisture concentrations (Table 6). One fertilizer, 30-0-0-0, increased yield $0.11 \mathrm{Mg} \mathrm{ha}^{-1}$ over the non-treated control. Sole liquid $\mathrm{N}$ sources (30-0-0-0 and $25-0-0-0,0.01 \%$ $\mathrm{Cl})$ had the highest overall yields at Albany. Although no fertilizer showed a significant yield reduction compared to the non-treated control, producers should avoid applying foliar fertilizers such as $0-0-30-0$ at the rates this study evaluated, which caused injury and plant necrosis. With the timing of application at tasseling, plants may not be able to recuperate from injury as well as younger plants. 


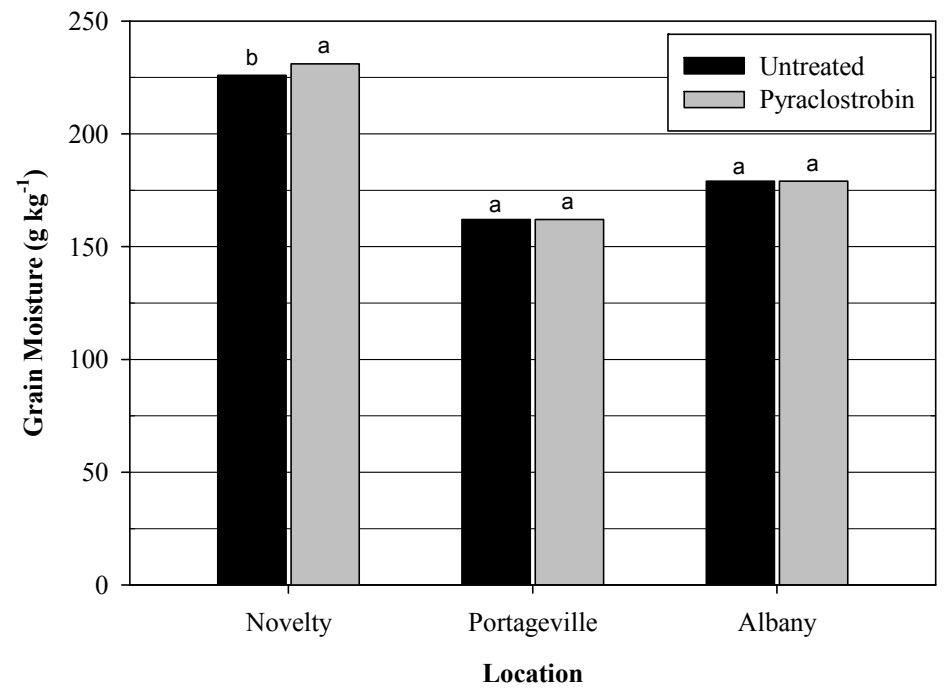

Figure 4. The effect of pyraclostrobin on harvested grain moisture at Novelty, Portageville, and Albany. Data were averaged over foliar fertilizer treatment and years

\section{Conclusions}

This study and previous research show that pyraclostrobin and other strobilurin fungicides interact with plants in multiple ways. Whether there is one interaction or many is unclear, but some factors such as the presence of pyraclostrobin lead to grain yield increases in corn crops over non-treated crops without visual differences in disease severity. During this study's six site-years, no differences appeared in disease severity among any treatments or controls. This may be a result of low levels of pathogenic inoculum, but is more likely a product of corn hybrid resistance to present pathogens. Of the 13 foliar fertilizers evaluated, 0-0-30-0 consistently caused injury over all six site-years. Ear leaf concentrations of nutrients varied widely over all macro- and micro-nutrients and site-years. Although many significant responses occurred from both main effects of pyraclostrobin and foliar fertilizers, few responses were consistent over all site-years, and no foliar fertilizer was responsible for more than one site-year's increase, except with regard to ear leaf S concentrations. Fertilizers, 5-0-20-13 and 0-0-25-17, both of which contain S, showed limited consistency in ear leaf nutrient concentrations seven DAT.

Neither pyraclostrobin nor any foliar fertilizer influenced grain protein concentrations at any of the site-years. Pyraclostrobin increased grain moisture and oil concentration at Novelty. Generally, pyraclostrobin had little effect on grain quality characteristics. Pyraclostrobin increased grain yields $0.5 \mathrm{Mg} \mathrm{ha}^{-1}$ at Novelty and Portageville, but showed no effect at Albany. An application of 30-0-0-0 was the only foliar fertilizer that significantly increased grain yields compared to the non-treated control. Sole N sources $(30-0-0-0$ and $25-0-0-0$, $0.01 \% \mathrm{Cl}$ ) were the two highest yielding treatments at Albany. The combination of increases in yield from pyraclostrobin and 30-0-0-0 may provide tank mixture options for corn produced in high-yielding environments.

\section{References}

Allison, J. C. S., \& Watson, D. J. (1966). The production and distribution of dry matter in maize after flowering. Ann. of Bot., 30, 365-381.

Bartlett, D. W., Clough, J. M., Godwin, J. R., Hall, A. A., Hamer, M., \& Parr-Dobrzanski, B. (2002). Review: The strobilurin fungicides. Pest Manag. Sci., 58, 649-662. http://dx.doi.org/10.1002/ps.520

BASF. (2010). Headline. BASF, Research Triangle Park, NC. Verified June 21, 2011. Retrieved November 2, 2009, from http://agproducts.basf.us/app/cdms

Bradley, C. A., \& Ames, K. A. (2010). Effect of foliar fungicides on corn with simulated hail damage. Plant Dis., 94, 83-86. http://dx.doi.org/10.1094/pdis-94-1-0083

Brennan, R. F. (1992). The role of manganese and nitrogen nutrition in the susceptibility of wheat plants to take-all in Western Australia. Fertilizer Research. Int. J. Fert. Use Technol., 31, 35-41. http://dx.doi.org/10.1007/bf01064225 
Brown, J. A., \& Jackson, R. K. (1955). A note on the potentiometric determination of chloride. Am. Soc. Hoc. Proc., 65, 187.

Butzen, S., Beudot, F., \& McInnes, B. (2005). Asian soybean rust: Fungicides. Crop Insights (Vol. 15, p. 2). Pioneer Hi-Bred Int., Inc.

Curley, S. (1994). Foliar nutrition. Midwest Laboratories Publication.

Fernández, V., \& Eichert, T. (2009). Uptake of hydrophilic solutes through plant leaves: Current state of knowledge and perspectives of foliar fertilization. Critical Reviews in Plant Sciences, 28, 36-68. http://dx.doi.org/10.1080/07352680902743069

Fernández, V., Del Rìo, V., Abadìa, J., \& Abadìa, A. (2006). Foliar iron fertilization of peach (Prunus persica (L.) Batsch): Effects of iron compounds, surfactants and other adjuvants. Plant Soil, 289, 239-252. http://dx.doi.org/10.1007/s11104-006-9132-1

Fixen, P. E., Snyder, C. S., Reetz, Jr., H. F., Yamada, T., \& Murrell, T. S. (2004). Nutrient management of soybeans with the potential for Asian rust infection. Potash \& Phosphate Institute, (PPI), Norcross, GA.

Gamble, P. E., \& Emino, E. R. (1987). Morphological and anatomical characterization of leaf burn in corn $\begin{array}{llllll}\text { induced from } \quad \text { foliar-applied nitrogen. } & \text { Agron. } & \text { J., } & 79, & 92-96 .\end{array}$ http://dx.doi.org/10.2134/agronj1987.00021962007900010019x

Glaab, J., \& Kaiser, W. M. (1998). Increased nitrate reductase activity in leaf tissue after application of the fungicide kresoxim-methyl. Planta, 207, 442-448. http://dx.doi.org/10.1007/s004250050503

Grossmann, K., \& Retzlaff, K. (1997). Bioregulatory effects of the fungicidal strobilurin kresoxim-methyl in $\begin{array}{lllll}\text { wheat } \quad \text { (Triticum } & \text { aestivum). }\end{array}$ http://dx.doi.org/10.1002/(sici)1096-9063(199705)50:1<11::aid-ps556>3.0.co;2-8

Grossmann, K., Kwaltowski, J., \& Caspar, G. (1999). Regulation of phytohormone levels, leaf senescence, and transpiration by the strobilurin kresoxim-methyl in wheat (Triticum aestivum). J. Plant Physiol., 154, 805. http://dx.doi.org/10.1016/s0176-1617(99)80262-4

Henggler, J. (2008). University of Missouri Extension. Retrieved May 25, 2009, from http://agebb.missouri.edu/irrigate/woodruff

Johnson, R. S., Rosecrance, R., Weinbaum, S., Andris, H., \& Wang, J. (2001). Can we approach complete dependence on foliar-applied urea nitrogen in an early-maturing peach? J. Amer. Soc. Hort. Sci., 126, 364-370.

Keeney, D. R., \& Nelson, D. W. (1982). Nitrogen-inorganic forms in methods of soil analysis. In A. L. Page, R. H. Miller \& D. R. Keeney (Eds.), Chemical and Microbiological Properties (2nd ed., Part 2, pp. 643-698). Am. Soc. of Agron. Inc. and Soil Sci. Soc. Am., Inc. Madison, WI.

Kettlewell, P. S., Cook, J. W., \& Parry, D. W. (2000). Evidence for an osmotic mechanism in the control of powdery mildew disease of wheat by foliar-applied potassium chloride. Eur. J. of Plant Path., 106, 297-300. http://dx.doi.org/10.1023/a:1008761202455

Köhle, H., Grossmann, K., Jabs, T., Gerhard, M., Kaiser, W., Glaab, J., ... Herms, S. (2002). Physiological effects of the strobilurin fungicide F 500 on plants. In H. W. Dehne, U. Gisi, K. H. Kuck, P. E. Russell \& H. Lyr (Eds.), Modern Fungicides and Antifungal Compounds III (pp. 61-74). Bonn: Mann GmbH \& Co. KG.

Kuehl, R. O. (1994). Statistical principles of research design and analysis. Duxbury Press, Belmont, CA.

Laverty, J. C. (1963). A modified procedure for the determination of phosphorus soil extracts. Soil Sci. Soc. Am. Proc., 27, 360-361. http://dx.doi.org/10.2136/sssaj1963.03615995002700030043x

Marschner, H. (1995). Mineral Nutrition of Higher Plants (pp. 229-312). Academic Press Limited, London, UK. http://dx.doi.org/10.1016/B978-012473542-2/50010-9

Mills, H., \& Jones, J. (1996). Plant analysis handbook II. MicroMacro Publ., Athens, GA.

Munkvold, G. P., Martinson, C. A., Shriver, J. M., \& Dixon, P. M. (2001). Probabilities for profitable fungicide use against gray leaf spot in hybrid maize. Phytopathology, 91, 477-484. http://dx.doi.org/10.1094/phyto.2001.91.5.477

Nachurs Alpine Solutions. (2009). Double-OK. Retrieved January 24, 2011, from http://www.nachurs.com/fertilizer/msds-sheets/0-0-30-msds.pdf 
Nason, M. A., Farrar, J., \& Bartlett, D. (2007). Strobilurin fungicides induce changes in photosynthetic gas exchange that do not improve water use efficiency of plants grown under conditions of water stress. Pest Manag. Sci., 63, 1191-1200. http://dx.doi.org/10.1002/ps.1443

Nelson, K. A., Motavalli, P. P., Stevens, W. E., Dunn, D., \& Meinhardt, C. G. (2010). Soybean response to preplant and foliar-applied potassium chloride with strobilurin fungicides. Agron. J., 102, 1657-1663. http://dx.doi.org/10.2134/agronj2010.0065

Nelson, K. A., Motavalli, P. P., Stevens, W. E., Kendig, J. A., Dunn, D., \& Nathan, M. (2012). Foliar potassium fertilizer additives affect soybean response and weed control with glyphosate. Intl. J. Agron. http://dx.doi.org/10.1155/2012/461894

Neumann, P. M., \& Prinz, R. (1975). The reduction by surfactants of leaf burn resulting from foliar sprays and a salt-induced inhibition of the effect. J. Sci. Food. Agric., 26, 909-914. http://dx.doi.org/10.1002/jsfa.2740260706

Newman, M. A. (2009). Effect of strobilurin fungicides and host resistance for control of gray leafspot of corn. Phytopathology, 99, S93.

Oerke, E. C. (2006). Crop losses to pests. J. of Agri. Sci., 144, 31-43. http://dx.doi.org/10.1017/s0021859605005708

Oerke, E. C., Beck, C., \& Dehne, H. W. (2001). Physiological effects of strobilurins on wheat yield. Phytopathology, 91, 67-71.

Parr, A. J., \& Loughman, B. C. (1983). Boron and membrane function in plants. In D. A. Robb \& W. S. Pierpoint (Eds.), Metals and micronutrients: Uptake and utilization by plants (pp. 87-107). Academic Press London. http://dx.doi.org/10.1016/B978-0-12-589580-4.50012-2

Poland J. A., Balint-Kurti, P. J., Wisser, R. J., Pratt, R. C., \& Nelson, R. J. (2009). Shades of gray: The world of quantitative disease resistance. Trends Plant Sci., 14, 21-29. http://dx.doi.org/10.1016/j.tplants.2008.10.006

Ritchie, S. W., Hanway, J. J., \& Benson, G. O. (1993). How a corn plant develops (p. 21). Iowa State Coop. Ext. Serv. Spec. Rep. 48. Ames, IA.

Rupe, J. C., Widick, J. D., Sabbe, W. E., Robbins, R. T., \& Becton, C. B. (2000). Effect of chloride and soybean cultivar on yield and the development of sudden death syndrome, soybean cyst nematode, and southern blight. Plant Dis., 84, 669-674. http://dx.doi.org/10.1094/pdis.2000.84.6.669

Ruske, R. E., Gooding, M. J., \& Jones, S. A. (2003). The effects of adding picoxystrobin, azoxystrobin and nitrogen to a triazole programme on disease control, flag leaf senescence, yield and grain quality of winter wheat. Crop Protection, 22, 975-987. http://dx.doi.org/10.1016/s0261-2194(03)00113-3

Sanogo, S., \& Yang, X. B. (2001). Relation of sand content, pH, and potassium and phosphorus nutrition to the development of sudden death syndrome in soybean. Can. J. Plant Pathol., 23, 174-180. http://dx.doi.org/10.1080/07060660109506927

SAS Institute. (2010). SAS user's guide. SAS Inst., Cary, NC.

Sweeney, D. W., Granade, G. V., Eversmeyer, M. G., \& Whitney, D. A. (2000). Phosphorus, potassium, chloride, and fungicide effects on wheat yield and leaf rust severity. J. Plant Nutr., 23, 1267-1281. http://dx.doi.org/10.1080/01904160009382099

Sweets, L. E., \& Wright, S. (2008). Integrated Pest Management: Corn Diseases. University of Missouri Extension.

Taiz, L., \& Zeiger, E. (2006). Growth and development, and auxin: The growth hormone. Plant Physiology (pp. 377-412; pp. 467-504).

Thomason, W. E., Wynn, K. J., Freeman, K. W., Lukina, E. V., Mullen, R. W., Johnson, G. V., ... Raun, W. R. (2001). Effect of chloride fertilizers and lime on wheat grain yield and take-all disease. J. Plant Nutr., 24, 683-692. http://dx.doi.org/10.1081/pln-100103662

Venancio, W. S., Rodrigues, M. A. T., Begliomini, E., \& de Souza, N. L. (2003). Physiological effects of strobilurin fungicides on plants. Publ. UEPG Ci. Exatas Terra, Ci. Agr. Eng., Ponta Grossa, 9, 59-68.

Ward, J. M. J., \& Nowell, D. C. (1998). Integrated management for the control of maize gray leaf spot. Integrated Pest Management Reviews, 3, 1-12. http://dx.doi.org/10.1023/A:1009694632036

Ward, J. M. J., Stromberg, E. L., Nowell, D. C., \& Nutter, Jr. F. W. (1999). Gray leaf spot: A disease of global 
importance in maize production. Plant Dis., 83, 884-895. http://dx.doi.org/10.1094/pdis.1999.83.10.884

Ypema, H. L., \& Gold, R. E. (1999). Kresoxim-Methyl: Modification of a naturally occurring compound to produce a new fungicide. Plant Disease, 83, 4-19. http://dx.doi.org/10.1094/pdis.1999.83.1.4

\section{Copyrights}

Copyright for this article is retained by the author(s), with first publication rights granted to the journal.

This is an open-access article distributed under the terms and conditions of the Creative Commons Attribution license (http://creativecommons.org/licenses/by/3.0/). 